PROCEEDINGS OF THE

AMERICAN MATHEMATICAL SOCIETY

Volume 137, Number 3, March 2009, Pages 1149-1152

S 0002-9939(08)09605-6

Article electronically published on September 17, 2008

\title{
A SHORT PROOF OF NONHOMOGENEITY OF THE PSEUDO-CIRCLE
}

\author{
KRYSTYNA KUPERBERG AND KEVIN GAMMON \\ (Communicated by Alexander N. Dranishnikov) \\ Dedicated to James T. Rogers, Jr., on the occasion of his 65th birthday
}

\begin{abstract}
The pseudo-circle is known to be nonhomogeneous. The original proofs of this fact were discovered independently by L. Fearnley and J. T. Rogers, Jr. The purpose of this paper is to provide an alternative, very short proof based on a result of D. Bellamy and W. Lewis.
\end{abstract}

\section{INTRODUCTION}

A pseudo-arc is a hereditarily indecomposable, chainable continuum. In 1948, E. E. Moise [16] constructed a pseudo-arc as an indecomposable continuum homeomorphic to each of its subcontinua. Moise correctly conjectured that the hereditarily indecomposable continuum given by B. Knaster 11 in 1922 is a pseudo-arc. Also in 1948, R. H. Bing [2 proved that Moise's example is homogeneous. In 1951, Bing 3 proved that every hereditarily indecomposable chainable continuum is a pseudo-arc and that all pseudo-arcs are homeomorphic. In 1959, Bing [4 gave another characterization of the pseudo-arc: a homogeneous chainable continuum.

The history of many other aspects of the pseudo-arc can be found in survey papers by W. Lewis [14] and [15].

In 1951, Bing [3] described a pseudo-circle, a planar hereditarily indecomposable circularly chainable continuum which separates the plane and whose every proper subcontinuum is a pseudo-arc. It has been shown by L. Fearnley in [6] and J. T. Rogers, Jr., in [17] that the pseudo-circle is not homogeneous. Fearnley also proved that the pseudo-circle is unique ([5] and [7]). The fact that the pseudo-circle is not homogeneous also follows from more general theorems proved in [8, 10, 13, and [18].

This paper offers yet another, very short proof, a consequence of a result of D. Bellamy and W. Lewis 11. Similarly as in 18, an infinite covering space of a plane separating continuum is used.

Received by the editors March 7, 2008

2000 Mathematics Subject Classification. Primary 54F15, 54F50.

Key words and phrases. pseudo-circle, pseudo-arc, homogeneous, composant, indecomposable continuum.

(C)2008 American Mathematical Society 


\section{Preliminaries}

Throughout the paper, a continuum will refer to a nondegenerate compact and connected metric space. A continuum is indecomposable if it is not the union of two proper subcontinua. A continuum is hereditarily indecomposable if every subcontinuum is also indecomposable. For a point $a$ in $X$, the composant $K(a)$ of $a$ in $X$ is the union of all proper subcontinua of $X$ containing $a$. An indecomposable continuum contains uncountably many pairwise disjoint composants; see [12, Theorem 7, page 212 .

A topological space $X$ is homogeneous if for any two points in $X$ there is a homeomorphism of $X$ onto itself mapping one point onto the other.

Let $C$ denote the pseudo-circle. We may assume that $C$ is contained in a planar annulus $A$ in such a way that the winding number of each circular chain in the sequence of crooked circular chains defining $C$ is one. Any homeomorphism $h$ : $C \rightarrow C$ extends to a continuous map $f: A \rightarrow A$ of degree \pm 1 . (First extend $h$ to a map $\bar{U} \rightarrow A$ for some closed annular neighborhood $\bar{U}$ of $C$; then compose a retraction of $A$ onto $\bar{U}$ with this extension.)

Let $\widetilde{A}$ be the universal covering space of $A$ with projection $p$. For any $\widetilde{x} \in \widetilde{A}$ and $\widetilde{y} \in p^{-1}(f(p(\widetilde{x})))$ there is a map $\widetilde{f}$ such that the diagram

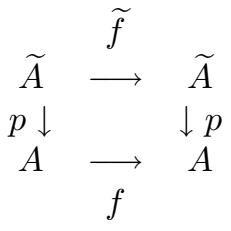

commutes and $\widetilde{f}(\widetilde{x})=\widetilde{y}$; see for example 9 , Theorem 16.3. Let $\widehat{A}$ be the disc that is a two-point compactification of $\widetilde{A}$. Denote the two added points of the compactification by $a$ and $b$. The map $\widetilde{f}$ extends uniquely to a map $F: \widehat{A} \rightarrow \widehat{A}$.

Let $\widetilde{C}=p^{-1}(C)$, and let $P=\widetilde{C} \cup\{a, b\}$, a two-point compactification of $p^{-1}(C)$. D. Bellamy and W. Lewis considered this set in [1] and proved that $P$ is a pseudoarc.

Denote by $H$ the restriction of $F$ to $P$ and note that

(1) either $H(a)=a$ and $H(b)=b$, or $H(a)=b$ and $H(b)=a$,

(2) $\widetilde{f}(\widetilde{C})=\widetilde{C}$ and hence $H(P)=P$,

(3) $\widetilde{f}_{\mid \widetilde{C}}$ is one-to-one.

Thus

Lemma. $H$ is a homeomorphism from $P$ to $P$.

\section{Proof of NONHOMOgEneITy of the PSEUdo-CIRCLE}

Theorem 1. The pseudo-circle is not homogeneous.

Proof. Let $K(a)$ and $K(b)$ be the composants of $a$ and $b$, respectively, in the pseudo$\operatorname{arc} P$. Let $\widetilde{x}$ and $\widetilde{y}$ be two points in $P$ such that $\widetilde{x} \in(K(a) \cup K(b))-\{a, b\}$ and $\widetilde{y} \in P-(K(a) \cup K(b))$. If $C$ were homogeneous, there would be a homeomorphism $h: C \rightarrow C$ taking $x=p(\widetilde{x})$ onto $y=p(\widetilde{y})$. Then there would be a homeomorphism $H: P \rightarrow P$ as described in section $[2$ taking $\widetilde{x}$ onto $\widetilde{y}$. This is not possible since under every such homeomorphism, the set $K(a) \cup K(b)$ is invariant; the image of a composant is a composant. 
Remark. It is not important for this proof that $K(a)$ and $K(b)$ are not the same set, but the authors are grateful to D. Bellamy and W. Lewis for showing that $K(a)$ and $K(b)$ were indeed different composants.

Theorem 2. If for some $x$, the composant $K(a)$ intersects the fiber $p^{-1}(x)$, then it contains $p^{-1}(x)$.

Proof. If $y \in p^{-1}(x) \cap K(a)$, then by the definition of a composant, there is a proper subcontinuum $W$ of $P$ that contains both $a$ and $y$. Let $g: \widetilde{C} \rightarrow \widetilde{C}$ be a deck transformation such that $p^{-1}(x)=\left\{g^{n}(y)\right\}_{n \in \mathbb{Z}}, \mathbb{Z}$ being the set of integers. Denote by $G$ the extension of $g$ to $P$. The set $W_{n}=G^{n}(W)$ is a continuum containing $a$ and $g^{n}(y)$. Thus $p^{-1}(x) \subset K(a)$.

Note that Theorem 2 and the remark above imply that $p(K(a)-\{a\}) \cap$ $p(K(b)-\{b\})=\emptyset$.

Question. Can the sets $p(K(a)-\{a\})$ and $p(K(b)-\{b\})$ be used to classify the composants of the pseudo-circle $C$ ?

\section{ACKNOWLEDGEMENTS}

The authors would like to thank Jim Rogers, David Bellamy, and Wayne Lewis for their comments.

\section{REFERENCES}

[1] D.P. Bellamy and W. Lewis, An orientation reversing homeomorphism of the plane with invariant pseudo-arc, Proc. Amer. Math. Soc. 114 (1992), 1145-1149. MR:1092915 (92g:54047)

[2] R.H. Bing, A homogeneous indecomposable plane continuum, Duke Math. J. 15 (1948), 729742. MR0027144(10:261a)

[3] R.H. Bing, Concerning hereditarily indecomposable continua, Pacific J. Math. 1 (1951), 43-51. MR0043451(13:265b)

[4] R.H. Bing, Each homogeneous nondegenerate chainable continuum is a pseudo-arc, Proc. Amer. Math. Soc. 10 (1959), 345-346 MR0105072 (21:3818)

[5] L. Fearnley, The pseudo-circle is unique, Bull. Amer. Math. Soc. 75 (1969), 398-401. MR0246265 (39:7569)

[6] L. Fearnley, The pseudo-circle is not homogeneous, Bull. Amer. Math. Soc. 75 (1969), 554558. MR0242126 (39:3460)

[7] L. Fearnley, The pseudo-circle is unique, Trans. Amer. Math. Soc. 149 (1970), 45-64. MR0261559(41:6172)

[8] C.L. Hagopian, The fixed-point property for almost chainable homogeneous continua, Illinois J. Math. 20 (1976), 650-652. MR0418057 (54:6101)

[9] S.T. Hu, Homotopy Theory, Academic Press, New York, 1959. MR0106454 (21:5186)

[10] J. Kennedy and J.T. Rogers, Jr., Orbits of the pseudocircle, Trans. Amer. Math. Soc. 296 (1986), 327-340. MR837815 (87g:54076)

[11] B. Knaster, Un continu dont tout sous-continu est indécomposable, Fund. Math. 3 (1922), 247-286.

[12] K. Kuratowski, Topology, Vol. II, Academic Press, 1968. MR.0259835 (41:4467)

[13] W. Lewis, Almost chainable homogeneous continua are chainable, Houston J. Math. 7 (1981), 373-377. MR640978 (83a:54044)

[14] W. Lewis, The pseudo-arc, Contemp. Math. 117, Amer. Math. Soc., Providence, RI, 1991, 103-123. MR:1112808 (92h:54046)

[15] W. Lewis, The pseudo-arc, Bol. Soc. Mat. Mexicana 5 (1999), 25-77. MR1692467 (2000f:54029)

[16] E.E. Moise, An indecomposable plane continuum which is homeomorphic to each of its nondegenerate subcontinua, Trans. Amer. Math. Soc. 63 (1948), 581-594. MR0025733 (10:56i) 
[17] J.T. Rogers, Jr., The pseudo-circle is not homogeneous, Trans. Amer. Math. Soc. 148 (1970), 417-428. MR.0256362 (41:1018)

[18] J.T. Rogers, Jr., Homogeneous, separating plane continua are decomposable, Michigan Math. J. 28 (1981), 317-322. MR629364 (83b:54045)

Department of Mathematics and Statistics, Auburn University, Auburn, Alabama 36849

E-mail address: kuperkm@auburn.edu

Department of Mathematics and Statistics, Auburn University, Auburn, Alabama 36849

E-mail address: gammokb@auburn.edu 